\title{
Lasing at the band edges of plasmonic lattices
}

\author{
A. Hinke Schokker and A. Femius Koenderink* \\ Center for Nanophotonics, FOM Institute AMOLF, Science Park 104, 1098 XG Amsterdam, The Netherlands \\ (Received 13 June 2014; revised manuscript received 24 September 2014; published 28 October 2014)
}

\begin{abstract}
We report room-temperature lasing in two-dimensional diffractive lattices of silver and gold plasmon particle arrays embedded in a dye-doped polymer that acts both as waveguide and gain medium. As compared to conventional dielectric distributed feedback (DFB) lasers, a central question is how the underlying band structure from which lasing emerges is modified by both the much stronger scattering and the disadvantageous loss of metal. We use spectrally resolved back-focal plane imaging to measure the wavelength and angle dependence of emission below and above threshold, thereby mapping the band structure. We find that, for silver particles, the band structure is strongly modified compared to dielectric reference DFB lasers since the strong scattering gives large stop gaps. In contrast, gold particles scatter weakly and absorb strongly, so that thresholds are higher, but the band structure is not strongly modified. The experimental findings are supported by finite element and Fourier modal method calculations of the single-particle scattering strength and lattice extinction.
\end{abstract}

DOI: 10.1103/PhysRevB.90.155452

PACS number(s): 73.20.Mf, 42.25.Fx, 42.55.Px, 42.55.Tv

In the past decade, plasmonics has become a very active field of research within optics owing to the unique opportunities for broadband strongly enhanced light-matter interaction in precisely fabricated metal nanostructures [1]. Enhanced light-matter interaction arises from the fact that plasmons, as hybrids of photons and charge density oscillations, are not restricted to the conventional diffraction limit. In addition, it has been shown that plasmon particles can enhance emission decay rates of fluorophores due to high Purcell factors over large bandwidths [2-6]. In fact, for low quantum efficiency fluorophores up to 1000-fold brightness enhancements per molecule have been reported near bowtie antennas [3-8]. Huge field enhancements (of order $10^{3}$ in electric field) have further been evidenced in surface-enhanced Raman and surfaceenhanced infrared spectroscopy $[9,10]$. Interest in exploiting plasmonics for lasing was sparked by the seminal paper by Bergman and Stockman in 2003 [11], where plasmonics was proposed for reaching deeply subdiffraction-sized lasers, ultralow thresholds, ultrafast laser dynamics, and unique properties due to the fact that only a few gain molecules and intracavity photons participate [12]. This vision of a "spaser" where lasing occurs due to nanoscale amplification of dark plasmons has led to a suite of recent experiments focusing on the smallest plasmonic lasers, targeting colloidal metal particles with gain [13] as well as hybrid plasmon modes confined in a narrow gap between a metal film and II-VI or III-V nanowires that provided the gain [14-19].

Aside from efforts to realize the highest possible field enhancements in narrow gaps of single structures, many efforts in plasmonics have been devoted to light-matter interaction in oligomers of scatterers and periodic lattices. Indeed, Yagi-Uda phased array antennas [20-24], Fano resonant oligomers [25-29], and periodic lattices [30-36] are among the most practical structures not only to control field enhancement, but also to obtain a balanced tradeoff between enhancement, Ohmic loss, and directivity control for emitters. In particular, in diffractive lattices, single-particle plasmon resonances can hybridize with Rayleigh anomalies or with planar waveguide

*f.koenderink@amolf.nl modes to form extended collective modes [37]. These systems have been shown to be very practical for improving broad area emission devices such as LEDs and phosphors, allowing simultaneous control over emission directivity and rate, at much lower losses than offered by single-particle resonances [30-33]. The picture that has emerged is that plasmonic structures can on one hand significantly enhance emission brightness from intrinsically very inefficient emitters by use of Purcell enhancement as a means to help radiative decay to outcompete nonradiative processes. On the other hand, in realistic application scenarios for solid-state lighting, already very efficient emitters can not benefit from plasmonics through Purcell enhancement, but do benefit through plasmonic band-structure effects that ensure redirection of light into select angles. In this case, the most efficient redirection is obtained through extended, not strongly localized, plasmon modes.

In the context of lasing, diffractive plasmon lattices were first studied by Stehr et al. [38], who reported a metallic particle grating based laser and showed linewidth narrowing and threshold behavior in these systems. A complementary geometry was reported very recently by van Beijnum et al. [39] who demonstrated a plasmon lattice laser based on hole arrays in gold paired to a III-V quantum well gain medium. Suh et al. and Zhou et al. [40,41], finally, reported on lasing in bowtie and nanodisk arrays, i.e., in particle arrays similar to those reported by Stehr et al. [38]. As in the case of spontaneous emission enhancement, plasmonic effects can impact lasing through two effects. On one hand, Purcell enhancements and near-field enhancement can accelerate emission dynamics. On the other hand, even in absence of strong Purcell enhancements, the formation of a plasmonic band structure with large stop gaps could modify the distributed feedback (DFB) mechanism. The work of Suh et al. and Zhou et al.[40,41] focused particularly on the role of plasmonic Purcell enhancements in lasing, for which reason an intrinsically very poor efficiency gain medium was chosen. Thereby, only the dye in very close proximity to the metal that experienced rate enhancement participated in the lasing. Here, we focus on the more application relevant scenario of plasmonic lasing in an efficient gain medium, in which case the main questions that arise are how the 
band structure of plasmonic lattice lasers differs from that of conventional two-dimensional (2D) distributed feedback lasers due to the plasmonic nature of its constituents, and how the tradeoff between much larger scattering strength and disadvantageously large loss of metal particles influences the lasing behavior.

In this paper, we report a comprehensive lasing study on particle array lasers fabricated from square lattices of silver $(\mathrm{Ag})$, gold $(\mathrm{Au})$, and as nonplasmonic reference titanium dioxide $\left(\mathrm{TiO}_{2}\right)$ embedded in a dye-doped polymer that at the same time acts as gain medium and supports a waveguide mode. We aim at uncovering what the band structure and lasing conditions of such systems are as a function of scattering strength (highest for $\mathrm{Ag}$ particles) and loss (highest for $\mathrm{Au}$ particles). Therefore, we use a high-efficiency dye as would be used in prospective solid-state applications, and operate in a regime where the effects of Purcell enhancements are expected to be small. To answer these questions, we have implemented a new measurement technique to map below-threshold emission and lasing in energy-momentum diagrams that can be acquired in a single shot in our subnanosecond optically pumped setup, and that span the entire angular collection range of a high-NA objective. We analyze the plasmonic band structure and for $\mathrm{Ag}$ arrays find stop-gap widths far in excess of those in dielectric DFB lasers and similar to those in the reported metal hole array laser of van Beijnum [39]. The paper is structured as follows. In Sec. I, we explain the setup, materials, and measurement procedure. In Sec. II, we present emission spectra, measured 2D Fourier space distributions of emission, and energy-momentum diagrams, all below and above threshold. In Sec. III, we interpret our band-structure measurements in terms of calculated single-particle scattering properties obtained from finite element simulations, and in terms of calculated angle-dependent extinction obtained with a rigorous coupled-wave analysis (RCWA) method.

\section{EXPERIMENT}

\section{A. Sample fabrication}

We use Menzel glass cover slides of $24 \times 24 \times 0.17 \mathrm{~mm}$ that have been cleaned in a solution of $\mathrm{H}_{2} \mathrm{O}, \mathrm{H}_{2} \mathrm{O}_{2}$, and $\mathrm{NH}_{4} \mathrm{OH}$ at $75^{\circ} \mathrm{C}$. After cleaning, we spincoat a positive resist to define our structures. For this we use the electron beam resist ZEP520 diluted in a ratio of 5:2 with anisole for which spincoating at $1500 \mathrm{rpm}$ results in a layer thickness of $150 \mathrm{~nm}$. With electron beam lithography we define hole arrays in a square pattern using dot exposures between 0.001 and $0.002 \mathrm{pC}$ using an electron gun voltage of $20 \mathrm{kV}$ and a current of $0.031 \mathrm{nA}$. We vary the lattice constant from 350 to $500 \mathrm{~nm}$ in steps of $10 \mathrm{~nm}$. The hole size is $100 \mathrm{~nm}$. The hole arrays are $200 \mu \mathrm{m}$ in size so that in optical experiments explained in the following, the arrays exceed the optical pump spot in diameter. To fabricate silver particle arrays we subsequently deposit $2 \mathrm{~nm}$ of chromium followed by $30 \mathrm{~nm}$ of silver by thermal evaporation, performed at a pressure of $<10^{-6}$ at an evaporation rate of $0.5-1 \AA / \mathrm{s}$. For the titanium dioxide samples, we directly deposit $30 \mathrm{~nm}$ of titanium dioxide using electron beam deposition. We perform liftoff by immersing the samples in $\mathrm{N}$-methylpyrrolidone (NMP) at $65^{\circ}$ for $5 \mathrm{~min}$. For
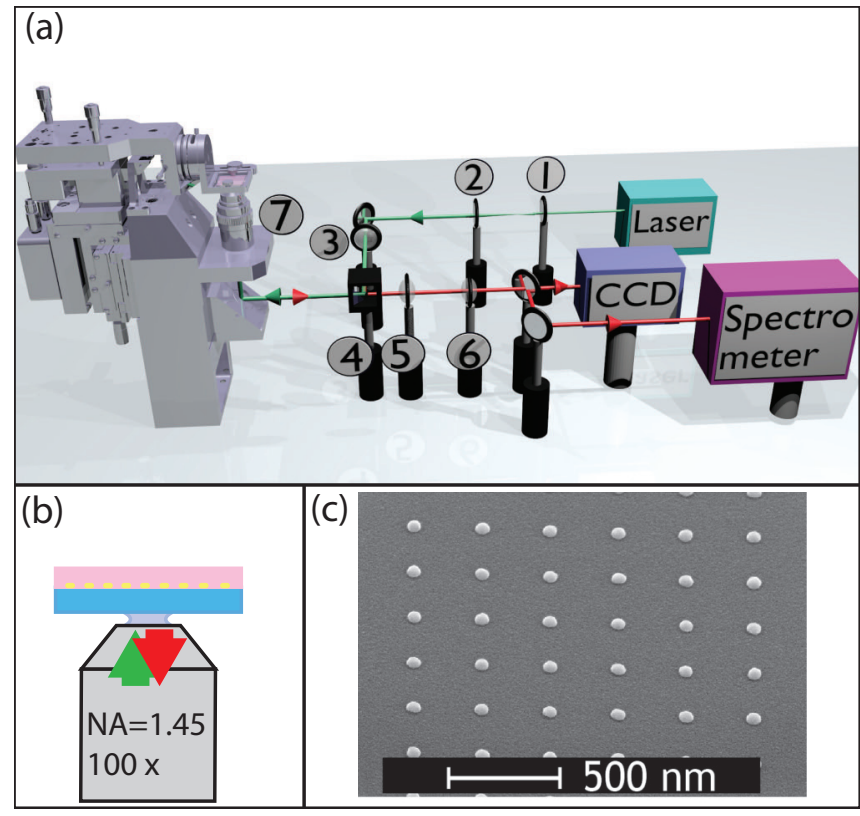

FIG. 1. (Color online) (a) Schematic of the setup. We illuminate the sample with laser light $(\lambda=532 \mathrm{~nm})$ from the glass side. We measure the fluorescence using the CCD and spectrometer. Right after the laser there are two lenses ( 1 and 2 ) that constitute a telescope to increase the beam diameter. After reflection off a mirror, there is an epilens (3) followed by a filter cube (4) that contains the dichroic mirror and a long pass filter, allowing only fluorescent red light on the detector side. On the right side of the filter cube there is a flippable Fourier lens $(f=200 \mathrm{~mm})(5)$ followed by a tube lens $(f=200 \mathrm{~mm})$ (6). The objective is mounted in a specially designed microscope mount (7). Not shown in the image are the AOM, together with a polarizer just in front of the telescope, to tune the laser power. In (b), we show a schematic of the objective with the sample. In (c), a scanning electron micrograph of one of the fabricated particle arrays is shown.

silver, liftoff is achieved by leaving them overnight at $50^{\circ}$ in anisole, as NMP degrades silver. After liftoff the samples are rinsed in isopropanol and blow dried with nitrogen. Figure 1(c) shows a Ag particle array resulting from the fabrication procedure.

To obtain a waveguide with gain, we use the negative photoresist SU8 and dope it with rhodamine $6 \mathrm{G}$ by mixing $5.25 \mathrm{mg}$ of Rh6G perchlorate with $1 \mathrm{~mL}$ of cyclopentanone (the solvent for SU8). The cyclopentanone with Rh6G is added to $1 \mathrm{ml}$ of SU8-2005, after which we ultrasonicate the solution for $10 \mathrm{~min}$. The final solution has a Rh6G perchlorate concentration of $0.25 \mathrm{wt}$. \%. We spincoat the SU8 solution on the particle array samples at $3000 \mathrm{rpm}$, resulting in a 450-nm-thick SU8 layer. This thickness results from a tradeoff between two requirements: on one hand sufficiently small thickness to ensure single-waveguide mode operation, and on the other hand sufficiently large thickness to ensure good mode overlap with the gain medium. After spincoating, we bake the samples for $2 \mathrm{~min}$ at $95^{\circ} \mathrm{C}$ to evaporate the excess cyclopentanone. Prepared as such, the SU8 is not cross linked, enabling removal of the SU8 layer with acetone after performing measurements on the samples. 


\section{B. Experimental setup}

We use an inverted fluorescence microscope as shown in Fig. 1(a). In this setup, the sample is mounted with the glass side down [close up sketch Fig. 1(b)], and both pump and detection occur through the objective, i.e., from the glass side. We use a home-built microscope tower, the most important pieces of which are an objective (Nikon, Plan Apo $\lambda 100 \times / 1.45$ NA) fixed to the microscope frame and a sample mount that can be translated in $X Y Z$ relative to the objective using micromechanical and piezo controls. The sample is pumped using a 532-nm pulsed laser (Teem Photonics, type STG-03E-1S0) which has a pulse width of $500 \mathrm{ps}$ and a maximum energy per pulse of $4.5 \mu \mathrm{J}$. We use an epilens in the pump path, resulting in a parallel beam with a diameter of $70 \mu \mathrm{m}$ emerging from the objective. The laser power is computer controlled by an acousto-optical modulator (AOM). We monitor the resulting pump power in real time with a home-built pulse integrator. To filter out unwanted reflected pump light, the fluorescence is sent through a long pass filter (Chroma, HHQ5451p) after passing the dichroic mirror (Semrock, Di01-R532-25x36). Fluorescence is detected by either a thermoelectrically cooled (Andor CLARA) Si CCD camera or a Shamrock303i spectrometer with an (Andor Ivac) Si CCD detector. To focus the light on the CCD and spectrometer entrance slit, we use an $f=200 \mathrm{~mm}$ tube lens. The pump laser can fire single pulses allowing single-shot measurements when triggering the laser, CCD, and spectrometer simultaneously. Single-shot exposure minimizes sample damage caused by bleaching of the Rh6G when performing a sequence of measurements for varying pump power. In addition to collecting images and spectra in real space, we do Fourier imaging by adding a lens on a flip mount at a focal distance from the back-focal plane of the objective [42-47].

Fourier imaging maps the back-focal plane of the objective onto the CCD camera, providing direct information on angular emission. The high NA objective $(\mathrm{NA}=1.45)$ allows for a large maximum collection angle of $\theta=73^{\circ}$ in glass, enabling us to image a large part of $k_{\|}$space. We note that the 2D back-focal plane images we collect in this fashion on the Clara CCD camera are panchromatic images, i.e., not separated in spectral components. Ideally, one would measure a spectrally resolved Fourier image since a spectrally resolved Fourier image would be a direct map of the dispersion diagram. This can be done by scanning a fiber which is coupled to a spectrometer through the entire Fourier image, or by imaging a slice of the Fourier image centered at $k_{x}=0$ onto the slit of an imaging spectrometer [48]. As we aim at single-shot measurements, the latter method is preferred. The Andor IVAC camera contains a CCD chip with $200 \times 1650$ pixels. To make spectral Fourier images, we set the spectrometer imaging mode to full imaging resulting in a full spectrum for 200 points along the $k_{y}$ axis.

For every particle array, we start by taking Fourier images of the fluorescence of single pump pulses as a function of input power by increasing the AOM voltage linearly in 200 steps from $0 \%$ to $50 \%$ of its maximum value. Subsequently, we flip in the mirror, sending the light to the spectrometer. We center the Fourier image on the spectrometer slit by fully opening the slit, observing the image in 0th order and moving the Fourier lens transversally until the circular Fourier image is in the center of the image of the slit. For Fourier spectra, we need to add the fluorescence resulting from 50 pulses because the light is spread over a large detector area. We find that 50 pump pulses do bleach the sample noticeably for higher pump powers. To make sure we see clear signs of lasing before the sample has bleached, we start with high pump powers (above lasing threshold) at an AOM percentage of $50 \%$ and go down in 200 steps to $0 \%$. From the Fourier spectra we calculate real-space spectra by integrating over $k_{y}$ for each wavelength. Because of bleaching, the threshold pump powers deduced from the Fourier images are slightly lower than the threshold we find from the Fourier spectra.

\section{RESULTS}

\section{A. Spectra}

Figure 2(a) shows a waterfall plot of spectra for increasing pump pulse power for a silver particle array with a pitch of

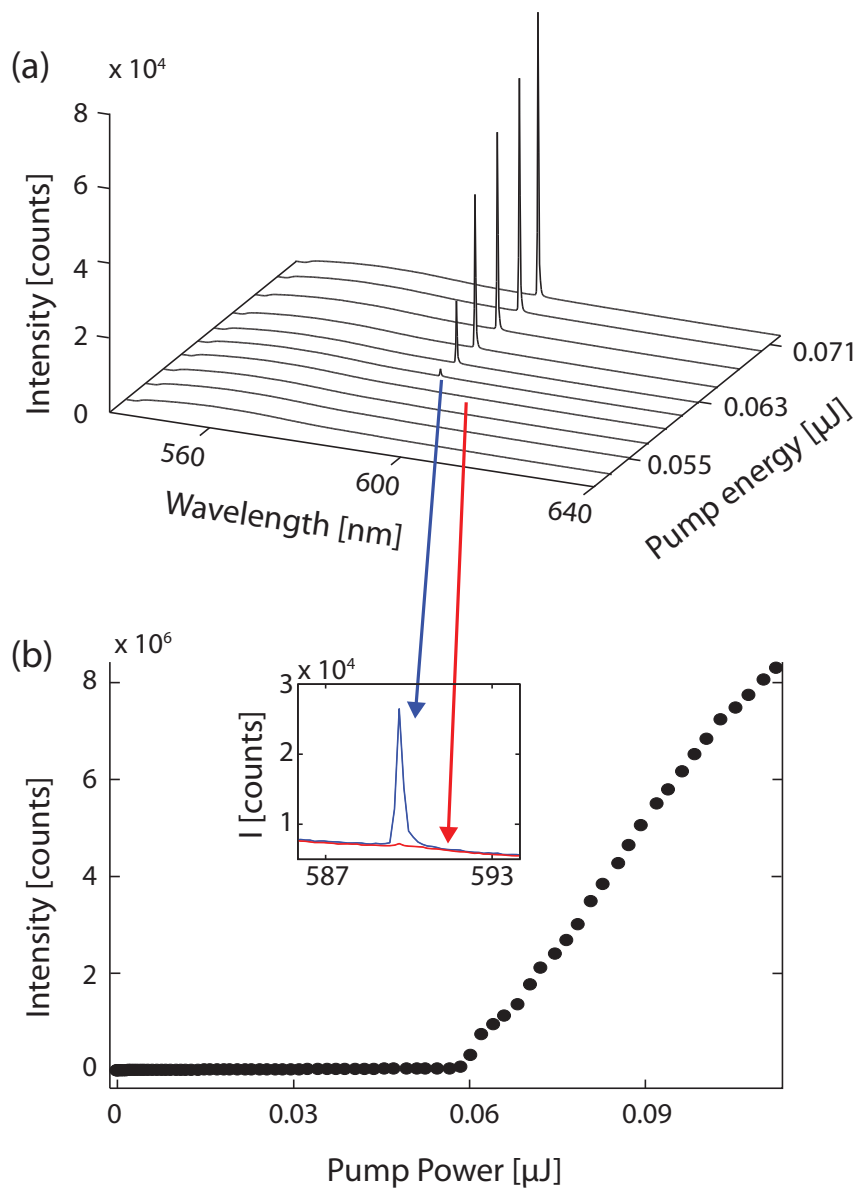

FIG. 2. (Color online) (a) Plot of emission spectra for different pump powers measured by imaging a Ag particle array (pitch $380 \mathrm{~nm}$, particle diameter $100 \mathrm{~nm}$ ) onto the spectrometer slit measured upon excitation with a single pump pulse. A clear threshold behavior can be seen from the sharp peak occurring for pump powers above $59 \mathrm{~nJ}$. Panel (b) threshold curve, plotting the area under the lasing peak versus pump pulse energy. The inset shows two spectra just above and just below threshold. 


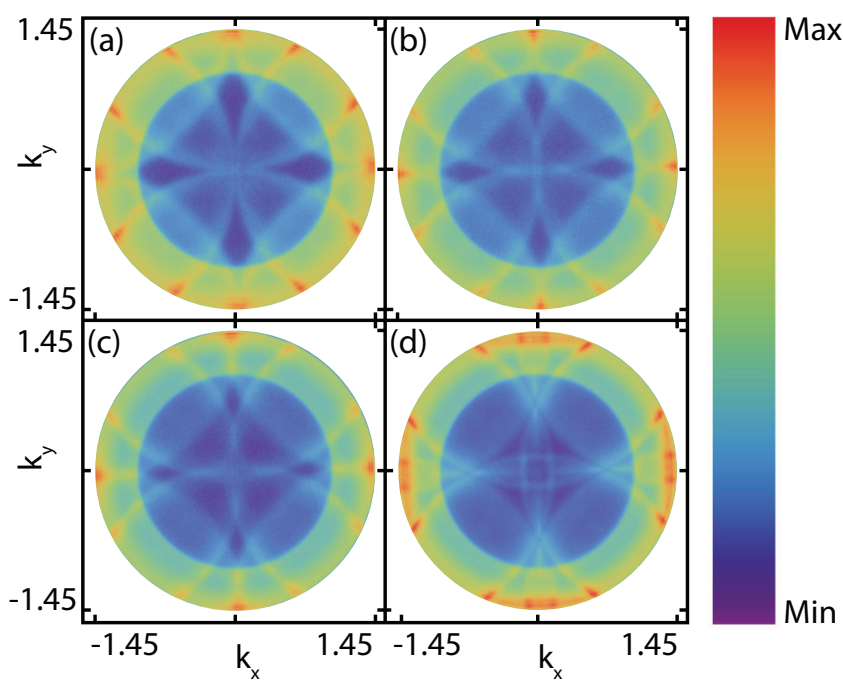

FIG. 3. (Color online) Fourier images below the lasing threshold for a pitch of (a) 360, (b) 370, (c) 380, and (d) $400 \mathrm{~nm}$. The color map ranges are [223, 1196], resp., [102, 739], [128, 941], and [319, 1948]. The reported wave vector axes are normalized to $\omega / c$.

$380 \mathrm{~nm}$ and particle diameter of $100 \mathrm{~nm}$. These spectra are obtained by applying full vertical binning over the central part of the Fourier image, thus including all angles along the $k_{y}$ axis. At a pump pulse energy of $59 \mathrm{~nJ}$, a clear peak emerges at a wavelength of $589 \mathrm{~nm}$ which dominates the emission spectrum for all higher pump powers. This can be seen more clearly from the inset of Fig. 2 where we plot a spectrum just below (red graph) and just above lasing threshold. The onset of the sharp peak is characteristic of lasing and the pump power at which it occurs is the lasing threshold. From the inset it can be seen that the lasing peak linewidth is on the order of a nanometer which is limited by the resolution of the spectrometer.

To construct a threshold curve, we define emission power as the total number of CCD counts under the lasing peak visible in Fig. 2(a), where we integrate over three spectral bins, corresponding to a total bandwidth of $0.5 \mathrm{~nm}$. Figure 2(b) shows the emission power versus pump power. The lasing threshold can be recognized by a sharp kink at a pulse energy just below $60 \mathrm{~nJ}$. The pulse energy density required to reach threshold is thus around $1.53 \mathrm{~mJ} / \mathrm{cm}^{2}$. This pulse energy density is comparable to that reported for plasmon particle arrays in a nonwaveguiding polymeric gain layer by Zhou et al. [41], although that laser operated much further into the infrared. These thresholds are approximately 10 times above those typically required for purely polymeric DFB lasers, such as the 2D MEH-PPV DFB laser reported by Turnbull [49]. Finally, we note that Stehr et al. [38] reported thresholds about equal to those of Turnbull et al. [49] for a gold particle array plasmon laser in a polyparaphenylene matrix. Aside from possible differences in gain coefficient, two possible explanations are the presence of Ohmic damping in the plasmonic particles and the much stronger outcoupling through scattering that plasmon particles offer.

In addition to measuring spectra as a function of pump power, we collect Fourier images of the fluorescence as shown in Figs. 3 and 4, which report Fourier images just below and

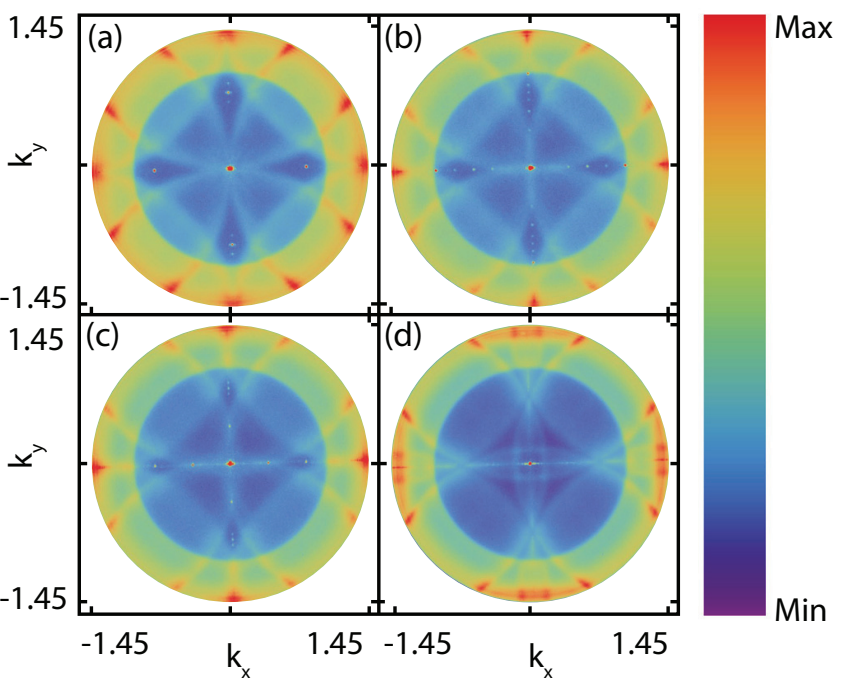

FIG. 4. (Color online) Fourier images just above the lasing threshold for a pitch of (a) 360, (b) 370, (c) 380, and (d) $400 \mathrm{~nm}$. The color map ranges are the same as in Fig. 3. Note the appearance of the narrow feature in the center of all images, which shows the onset of lasing emission. The reported wave vector axes are normalized to $\omega / c$.

just above threshold, respectively, for four particle lattices with pitches $d=360,370,380$, and $400 \mathrm{~nm}$ [panels (a)-(d) in both figures]. In each figure, two features stand out independent of particle pitch. First, we see a high-intensity ring where most of the emission exists, indicating that most below-threshold emission exits at large angles. The inner edge of this ring corresponds to an NA of 1 or, equivalently, to the critical angle of the glass-air interface. The outer edge is set by the NA of the immersion oil objective. That fluorescence is preferentially emitted at angles just above an NA = 1 is a well-known feature for emitters on a glass-air interface $[43,48,50]$, and consistent with radiation pattern calculations according to Chap. 10 of Ref. [50], which show radiation patterns peaking at the critical angle of the glass-air interface.

Second, we see higher-intensity circles, displaced from the center and repeating in the $k_{x}$ and $k_{y}$ directions with a fixed period that changes with particle pitch. Indeed, based on the wave-vector scale calibration of our images, we can confirm that the Fourier space periodicity corresponds to a square lattice with $\frac{2 \pi}{d}$ period, i.e., to the reciprocal lattice of our structure. Based on the absolute wave-vector scale calibration of our images, we can also convert the radius of the circles into a propagation constant. We find $k_{\text {circle }} /(\omega / c)=1.52 \pm 0.03$, where the factor 1.52 corresponds very well with the calculated mode index for the fundamental TE and TM guided mode of the SU8 layer as calculated from Eqs. (4.4) and (4.17) in Ref. [51].

Overall, the fluorescence Fourier pattern is a direct, singleshot CCD image of the repeated zone scheme isofrequency surface of the waveguide mode dispersion that is well known to occur for periodically corrugated waveguides [52-55]. In other words, due to in-plane Bragg scattering that couples any $\mathbf{k}_{\|}$into $\mathbf{k}_{\|}+\mathbf{G}$, the circular dispersion relation $\omega, \mathbf{k}_{\|}$ of the waveguide mode of index $n=1.52$ repeats at every 
reciprocal lattice point $\mathbf{G}$. We expect that for each intersection of circles, an anticrossing should be visible, as the finite scattering strength of the plasmon particles should open up noticeable stop gaps in the nearly free-photon dispersion approximation. However, any stop gaps that may occur at the crossing points are obscured in these images due to the fact that spectral averaging limits their sharpness. To overcome this problem, we use the spectral imaging procedure described in the experimental section which gives us full dispersion diagrams of the emission over the entire detectable $\mathbf{k}_{y}$ range. The resulting $\omega, k_{y}$ diagrams are shown in Figs. 5(a)-5(d), just below threshold and in Figs. 5(e)-5(f), just above threshold. Again, we can see high-intensity bands corresponding to the high-intensity ring in the Fourier image. In addition, we distinguish a pair of steep straight lines that cross at the $\Gamma$ point $\left(k_{y}=0\right)$ at a frequency of $3.4 \times 10^{15} \mathrm{rad} \cdot \mathrm{s}^{-1}$ for $d=360 \mathrm{~nm}$. Furthermore, a parabolic band with minimum at (or just above) the crossing of steep lines is evident, that has its minimum at or just above the crossing of steep lines. These features can be understood by looking at a generic dispersion diagram respresenting the folded free-photon dispersion, as indicated in Fig. 5(i). The straight lines that begin at the origin are the linear waveguide dispersion.

At the first-order Bragg condition $k_{y}=\frac{\pi}{d}$, the free-photon dispersion copies shifted along $k_{y}$ by $\frac{2 \pi}{d}$ fold back into the first Brillouin zone. At twice this frequency, the second-order diffraction condition is met, as is evident from the fact that the folded dispersions again cross (straight lines). For a square lattice at the same frequency, the diffraction condition is met for the grating vector $\mathbf{G}=\left(\frac{2 \pi}{d}, 0\right)$ perpendicular to the $k_{y}$ axis. This diffraction leads to the parabola. When the particle pitch increases, the first-order Bragg condition is met at a lower frequency and all features move down as the waveguide mode circles repeat with a smaller period. In Figs. 5(e)-5(h), we show Fourier spectra just above the lasing threshold. Lasing spots are visible as high-intensity spots that occur exactly at the crossing point of the lines with the parabola. This corresponds to the second-order Bragg diffraction condition. Qualitatively, this behavior is exactly as generally observed for 2D DFB lasers $[38,41,49]$. In Fig. 5(h), we can see that there is also lasing on the third-order Bragg condition, as the frequency for the third-order condition has moved down into the gain window of Rh6G for a pitch of $400 \mathrm{~nm}$. Polarization-dependent measurements reveal that the lasing mode is a TE mode, corresponding to what has been reported in literature for dielectric DFB lasers [56,57].

Broadly speaking, it appears that the Ag particle array laser is close to a standard DFB laser in that it operates at the lower edge of the second diffraction stop gap at $\mathbf{k}_{\|}=0$ [stop-gap edges indicated by white ticks in Fig. 5(f)]. We now ask how the plasmonic nature of the Ag particles modifies the DFB characteristics compared to a dielectric DFB laser [30,59]. In order to probe this question, we compare three systems, namely, (1) the Ag particle arrays, (2) arrays of the same pitch of dielectric $\mathrm{TiO}_{2}$ particles, and (3) Au particles that should show stronger absorption yet weaker scattering than the Ag particles. In Fig. 6, we compare the dispersion diagram for a DFB laser with silver scatterers with a DFB laser that uses $\mathrm{TiO}_{2}$ and $\mathrm{Au}$ particles, for a particle pitch of $380 \mathrm{~nm}$ and a particle size of 150 and $100 \mathrm{~nm}$, respectively. For $\mathrm{TiO}_{2}$, we used larger particles of 150-nm diameter, as the $\mathrm{TiO}_{2}$ particle arrays with particle sizes of $100 \mathrm{~nm}$ did not show lasing. This we assign to the weak scattering strength of $100 \mathrm{~nm} \mathrm{TiO}_{2}$ disks. Indeed, numerical analysis reported below of scattering cross sections show that $\mathrm{TiO}_{2}$ scatterers of the same volume have a scattering strength at least 10 times lower than metal particles. Two clear differences are visible between Figs. 6(c) and 6(d). For the $\mathrm{TiO}_{2}$ sample, the parabolic band appears to be a single feature, as expected from Fig. 5(c). However, strictly speaking the parabolic band is degenerate, originating from both the $(1,0)$ and $(-1,0)$ diffraction order. Remarkably, for the $\mathrm{Ag}$ particle array this degeneracy is distinctly split, pointing at the strong scattering strength of Ag particles. Figure 6(b) shows the measured dispersion diagram for an Au array. Evidently, the bands are broad, at least as much as in the Ag case, but not clearly split, as in the $\mathrm{TiO}_{2}$ case. This points at the higher loss, yet weak scattering strength at $590 \mathrm{~nm}$, of Au particles compared to Ag.

For dielectric photonic crystals, relative stop-gap width $\Delta \omega / \omega_{0}$ is frequently used as a dimensionless parameter to sort photonic crystals by their photonic interaction strength [60-62]. In that case, the relative stop-gap width is proportional to the ratio of scatterer polarizability to unit-cell volume. In real-space terms, the stop-gap width provides a direct measure for the Bragg length (number of lattice planes required for $1 / e$ diffraction efficiency), the crystal size needed to develop a significant local density of states (LDOS) suppression, and the crystal size required to achieve LDOS enhancement of mode density at a band edge of any significant magnitude and over significant bandwidth [60-62]. On this basis, we use the width of the stop gap to quantify differences in the dispersion diagram for different plasmonic laser systems. Figure 6(a) shows a plot of the relative size of this stop gap as a function of particle pitch for $\mathrm{Ag}, \mathrm{Au}$, and $\mathrm{TiO}_{2}$. The horizontal lines indicate relative stop-gap values reported by van Beijnum [39] (black), Turnbull [49] (purple), and Noda [58] (red) for a plasmonic hole array laser, a nonplasmonic DFB laser, and a photonic crystal band-edge laser in a $2 \mathrm{D}$ semiconductor membrane. The blue dots for Ag show that the relative band gaps are large compared to reported values for dielectric systems and approach the value reported for the plasmonic laser of van Beijnum [39]. The red triangles and green squares represent relative band gaps for $\mathrm{TiO}_{2}$ and $\mathrm{Au}$. For these two materials, the stop gaps are smaller than the width of the band and therefore are essentially zero. To conclude, the Ag particle array DFB lasers are markedly different from the nonplasmonic lasing systems, and due to the strong scattering the diffractive coupling in the dispersion relation is as strong as in the plasmonic hole array laser. Ideally, to verify if the correlation between stop-gap width and sample geometry is exclusively with scattering strength (or "polarizability," as in the photonic crystal case) one would need to independently vary physical particle volume at fixed optical volume (polarizability), or vice versa, in an otherwise fixed gain medium. Unfortunately, this will be difficult to realize: while our data clearly show that the nonplasmonic $\mathrm{TiO}_{2}$ particles of equal physical volume are so weakly scattering as to give neither stop gap nor lasing, a larger optical volume at fixed physical size than for the silver particles can not be realized in the gain window of our dye. Somewhat larger optical volume could be reached with either 

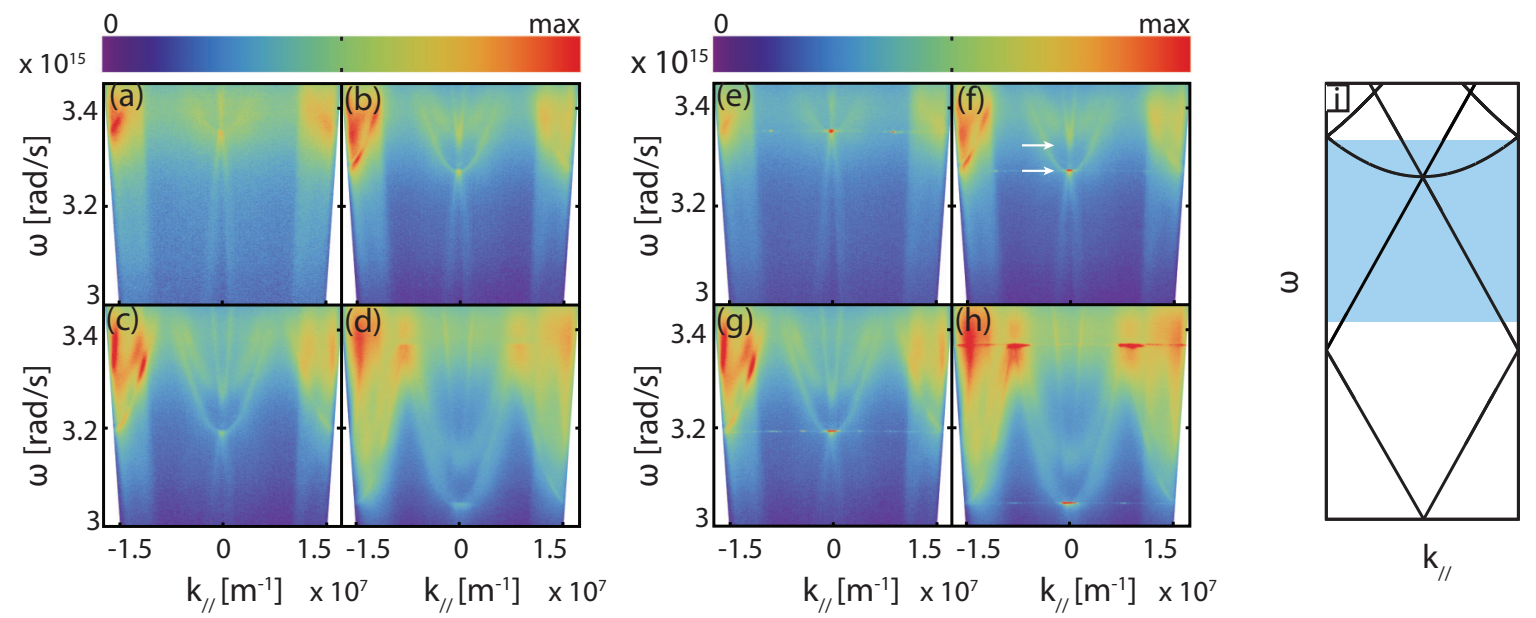

FIG. 5. (Color online) Fourier spectra for four different pitches, just below threshold (a)-(d) and just above threshold (e)-(h), and a schematic of a general band diagram (i). Measurements are for a pitch of $360 \mathrm{~nm}$ [(a) and (e)], $370 \mathrm{~nm}[(\mathrm{~b})$ and (f)], $380 \mathrm{~nm}[(\mathrm{c})$ and (g)], and $400 \mathrm{~nm}[(\mathrm{~d})$ and (h)]. The maximum values of the color bar are set at 230, 200, 200, and 250 counts, respectively, in (a)-(d). Above threshold, the maximum values of the color bar are 230, 250, 200, and 300 counts, respectively [panels (e)-(h)]. All color bars start at 0 . Note the lasing emission that appears as a narrow feature at $k_{\|}=0$ (the horizontal lines across the diagram, surrounding the lasing peak, are CCD blooming artifacts). For clarity, we have indicated the lower and upper stop band edges for second-order diffraction by white arrows in panel (f). Lasing occurs at the lower edge.

Ag or Au particles by increasing their size, however, only at strongly red-shifted resonance frequencies.

Finally, we note that in order to cross the lasing threshold, the Au samples typically require at least 10 times higher pump fluence $\left(0.066 \mathrm{~mJ} / \mathrm{cm}^{2}\right.$ versus $1.107 \mathrm{~mJ} / \mathrm{cm}^{2}$ for the example in Fig. 6). This finding is consistent with the much more advantageous Ohmic loss of $\mathrm{Ag}$ versus $\mathrm{Au}$, which for $\mathrm{Au}$ nanodisks means a much lower scattering strength and a much lower albedo. Regarding the comparison between thresholds of the $\mathrm{TiO}_{2}$ sample with the thresholds of Ag and Au samples, we have to note that a direct comparison is hampered by the fact that the much lower scattering strength of $\mathrm{TiO}_{2}$ disks means that much larger particles were required than for the plasmonic samples to reach the lasing transition at all. Generally, most all-dielectric samples that actually lased (particle diameters above $150 \mathrm{~nm}$ ) had lower thresholds than their plasmonic counterparts. This finding indicates that $\mathrm{TiO}_{2}$ offers low loss, yet also a much weaker per-particle cross section contributing to feedback.

\section{THEORY}

\section{A. Single-particle scattering}

We use COMSOL $4.3 \mathrm{~b}$ to determine the extinction cross sections for single particles. In Fig. 7, we plot the extinction cross section for $\mathrm{Ag}, \mathrm{Au}$, and $\mathrm{TiO}_{2}$ for two different incidence conditions. In Fig. 7(a), an $x$-polarized plane wave is incident along the $z$ direction (parallel to the symmetry axis of the particle disk). In Fig. 7(b), an $x$-polarized plane wave is incident along the $y$ axis. We used an index of $n=1.65$ for
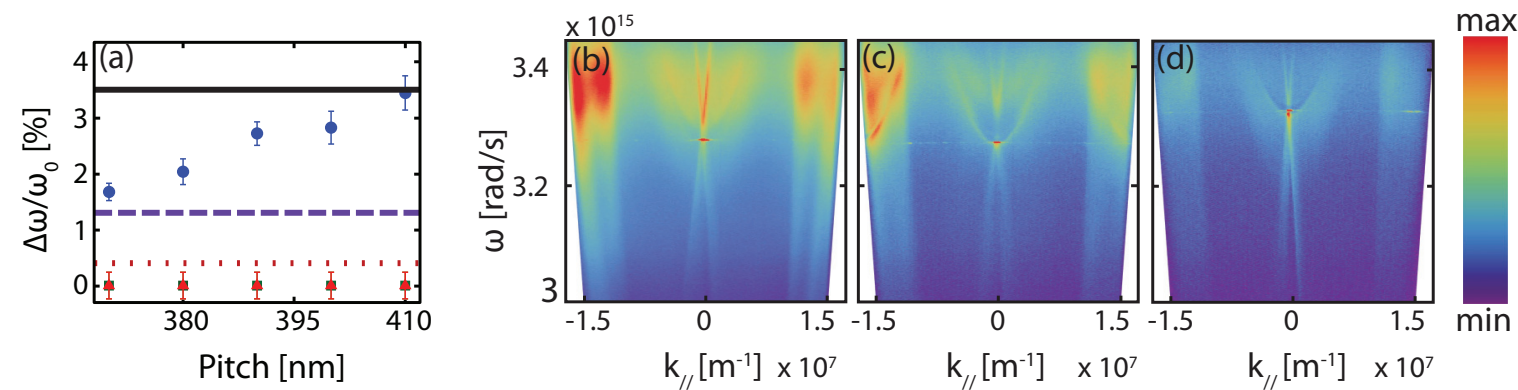

FIG. 6. (Color online) In (a) the relative size of the stop gap is plotted for $\mathrm{Ag}$ (blue dots), $\mathrm{Au}$ (red triangles), and $\mathrm{TiO}_{2}$ (green squares) together with literature values of van Beijnum [39] (black), Turnbull [49] (purple), and Noda [58] (red). In addition, fluorescence in the $\omega, k_{y}$ plane is plotted for $\mathrm{Au}(\mathrm{b}), \mathrm{Ag}$ (c), and $\mathrm{TiO}_{2}$ (d) for a pitch of $370 \mathrm{~nm}$. The maximum values of the color bar are 550, resp., 250 and 300 counts in panels (b)-(e), with color bars starting at 0 . The plots are made just above the lasing threshold, meaning pump powers differ from panel to panel. The pump powers are $944 \mathrm{~nJ}(\mathrm{Au}), 99.5 \mathrm{~nJ}(\mathrm{Ag})$, and $38 \mathrm{~nJ}\left(\mathrm{TiO}_{2}\right)$, respectively. The notably larger pump power for $\mathrm{Au}$ results in a much higher background fluorescence level as is visible for a frequency range centered at the the Rh6G emission peak in (b). 

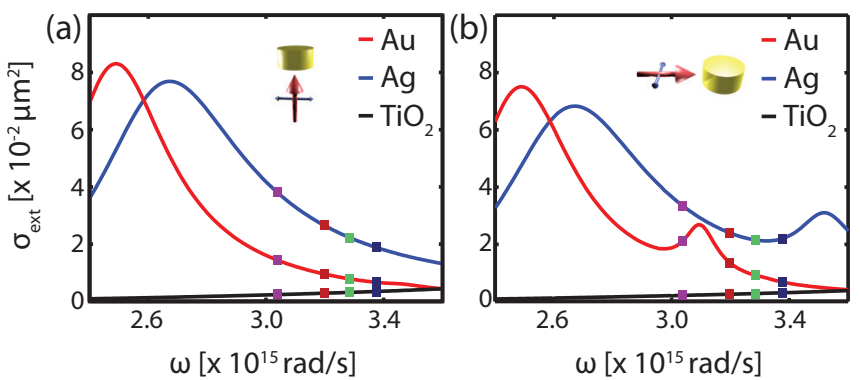

FIG. 7. (Color online) Extinction cross sections for $\mathrm{Ag}, \mathrm{Au}$, and $\mathrm{TiO}_{2}$ as a function of $\omega$ obtained using COMSOL. The $z$ axis is defined as the axis parallel to the symmetry axis of the particle disks. In plot (a) the plane wave is incident along the $z$ axis and in plot (b) the plane wave is incident from the side of the particle, with polarization in the plane of the particle. For clarity, the curves for $\mathrm{TiO}_{2}$ are scaled by a factor 10 as indicated.

SU8 as the surrounding medium. For the permittivity, we use a modified Drude model fitted to the optical constants of Johnson and Christy [63]:

$$
\epsilon_{r}=\epsilon_{\infty}-\frac{\omega_{p}^{2}}{\omega(\omega+i \gamma)} .
$$

For Au we use $\epsilon_{\infty}=9.54, \omega_{p}=1.35 \times 10^{16} \mathrm{rad} / \mathrm{s}$, and $\gamma=$ $1.25 \times 10^{14} \mathrm{rad} / \mathrm{s}$ and for Ag we use $\epsilon_{\infty}=5.43+0.55 i, \omega_{p}=$ $1.39 \times 10^{16} \mathrm{rad} / \mathrm{s}$, and $\gamma=8.21 \times 10^{13} \mathrm{rad} / \mathrm{s}$, as reported in Ref. [64].

Both $\mathrm{Ag}$ and $\mathrm{Au}$ exhibit a clear resonance which is completely absent for $\mathrm{TiO}_{2}$. In addition, one can see that the peak of Ag is blue-shifted with respect to the resonance peak of $\mathrm{Au}$. The lasing frequencies for the studied four particle pitches are indicated by the squares. For a plane wave along the $y$ direction we can distinguish two peaks, where the smallest peak (at higher frequencies) corresponds to the quadrupolar resonance. For incidence normal to the disks, as would be the case in transmission experiments that probe the sample under normal incidence, no quadrupole response is noticeable. However, for the distributed feedback in-plane scattering of the TE-polarized waveguide mode is important. This distributed feedback hence might benefit from the quadrupole response for enhanced scattering and near fields.

\section{B. Band structure}

Finally, we have calculated the band structures of the plasmon gratings embedded in the waveguide structures as they would appear in extinction, using rigorous coupled-wave analysis (RCWA) that is optimized for 2D periodic and stratified problems. In particular, we have used the freely available implementation " $S$ " (github version 1.0.0) by Liu and Fan [65] of the Fourier modal method developed by Li $[66,67]$, that uses the appropriate factorization rules for high-index contrast gratings. While convergence can be notoriously poor for metallic gratings, we found excellent convergence when using parallelogrammic truncation. We used a truncation to 361 plane waves. We set $n_{\mathrm{SU}}=1.65$ and take the particle sizes and dielectric constants as in COMSOL. The index of the glass substrate is set to $n=1.51$, while we take as

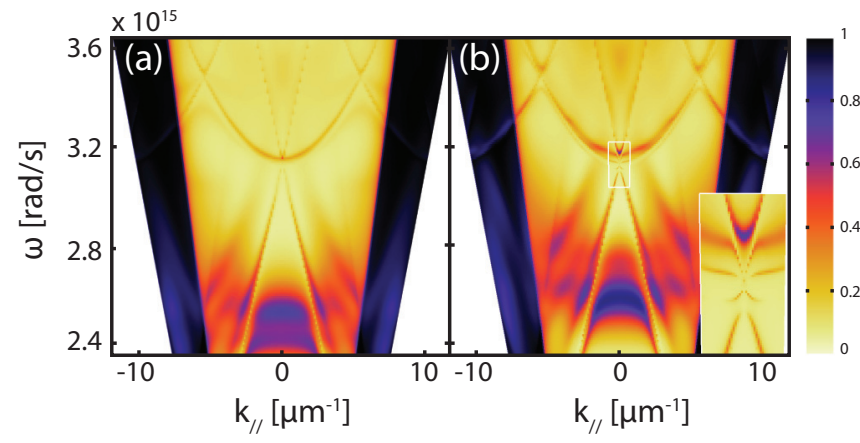

FIG. 8. (Color online) Extinction as a function of $\mathrm{k}_{\|}$and $\omega$ for gold (a) and silver (b). Evident for both diagrams are (1) the highextinction region for lower $\omega$, corresponding to the single-particle resonance, and (2) the generic folded band diagram features (lines and parabolas). For silver, a clear stop gap is visible at the $\Gamma$ point $\left(\mathbf{k}_{\|}=0\right)$ that is not apparent in the diagram for gold. The inset shows a zoom-in of this region, highlighting the intricate anticrossing with the two parabolic bands.

waveguide thickness $450 \mathrm{~nm}$. Again, we use the Drude model to describe the permittivity of $\mathrm{Ag}$ and $\mathrm{Au}$. We obtain extinction as a function of incidence angle, resulting in the extinction dispersion diagrams shown in Fig. 8. While the Fourier modal method is a fully vectorial method that takes coupling between Bloch harmonics of all polarizations into account, here we report specifically on extinction in the case of $s$-polarized incidence, corresponding to coupling to TE-waveguide modes. This choice is motivated by our observation of the polarization of the lasing mode, and is commensurate with 2D DFB lasing in dielectric structures [56,57]. Both the Ag and Au lattices show a band of high extinction at $k_{\|}=0$ close to $\omega=2.5 \times$ $10^{15} \mathrm{rad} / \mathrm{s}$, where as expected the gold array is red-shifted compared to the Ag array. This extinction band corresponds to the single-particle dipole resonance. Compared to Fig. 7, Fig. 8 shows single-particle resonances slightly red-shifted as in $S^{4}$ simulations infinite particle arrays are considered. When the interparticle distance is comparable to the wavelength, longitudinal coupling between the individual dipole scatterers is known to cause the observed red-shift [68]. The higher extinction due to the single-particle resonance couples to the waveguide mode [59], leading to anticrossings at $k_{\|}$along the straight lines corresponding to the backfolded waveguide mode dispersion. This anticrossing was observed experimentally by Rodriguez et al. [32]. At $3.2 \times 10^{15} \mathrm{rad} / \mathrm{s}$ and above, there are the expected parabola and straight lines from the folded free-photon dispersion discussed in the experimental results. In this part, we can see two clear differences between $\mathrm{Au}$ and Ag. First, the parabola for silver is broader than that of gold. Second, at the crossing point of the parabola with the straight lines a clear avoided crossing can be seen for silver, whereas for gold the $\Gamma$ point does not show a gap and corresponds with the folded free-photon dispersion. The complex avoided crossing that lifts the degeneracy between the two parabolas for silver can be seen more closely in the inset in Fig. 8(b). While the single-particle resonance is broad $(Q=4.4)$, the hybrid modes resulting from coupling of waveguide mode and plasmon have $Q>150$, i.e., damping much less than the single-particle radiative damping. 
Returning to a comparison with the measurements, we conclude that the theory reproduces all the salient features. For the gold particle lattices, the scattering strength per particle is low. Consequently, in both theory and data, the stop-gap width is small. For the silver particle lattice, however, the scattering strength per particle is much higher and consequently in both theory and experiment a clear stop gap opens up at the second-order diffraction, and the degenerate parabolas split, and broaden. Based on the single-particle response, we surmise that the precise coupling strength that splits the bands at $3.2 \times 10^{15} \mathrm{rad} / \mathrm{s}$ and above is dependent on the quadrupole response. Finally, we note that we also calculated dispersion for $\mathrm{TiO}_{2}$ particle lattices. As in experiment, the calculated dispersion (not shown) only shows narrow features that essentially coincide with the folded SU8 waveguide dispersion.

\section{CONCLUSION AND OUTLOOK}

Overall, it is remarkable that despite their loss, silver particle array lasers provide lasing characteristics reasonably competitive with dielectric DFB systems, commensurate with the established notion that the extended diffractive modes that plasmon arrays support well off their individual resonance frequencies are promising for balancing loss and light-matter interaction strength. Furthermore, our results and experimental methods open up many questions for further study. To start, it would be very interesting to sweep the diffraction condition and gain window onto the plasmon resonance frequency. This allows us to continuously trace how lasing occurs along the transition from a weakly coupled plasmon-waveguide hybrid system to a purely plasmonic mode. Second, if one could probe lasing in a given system for gain media of different quantum efficiencies, one could further clarify the role of high Purcell factors near metal particles in lasing plasmonic lattices. In our work, we estimate that less than $1 \%$ of emitters are within $20 \mathrm{~nm}$ of metal, i.e., are in a position where Purcell enhancement might occur. Since we use an intrinsically already highly efficient, bright emitter, there is no measurable Purcell enhancement, and in fact those dyes that experience rate enhancement likely are rendered less efficient contributors to the lasing process due to quenching. This should be contrasted to the work of Suh et al. and Zhou et al. [40,41]. Third, by significantly reducing the pitch, one enters the "lasing spaser" regime proposed by Zheludev et al. Zheludev et al. [69] proposed that when plasmon resonators with gain are arrayed with pitch much smaller than the wavelength, lasing will not occur on a diffraction condition, yet coherence will be established to give lasing emission normal to the lattice plane. Generally, we expect that if a dense metasurface would lase, it would do so on the lowest loss mode in the wave-vector diagram. Hence, this regime directly necessitates a deep study of the dispersion relation of collective modes in metamaterials. Finally, an obvious extension of our work is to study aperiodic systems [70-72]. Previous studies have shown that aperiodic and quasiperiodic systems, as an intermediate state between order and disorder, have modes that are neither Bloch states as in a periodic lattice, nor exponentially localized states as in a random array, but exhibit "critical modes" that show strong spatial fluctuations in field amplitude [70,73]. It would be interesting to study these critical modes in the context of lasing.

\section{ACKNOWLEDGMENTS}

We thank M. Kamp, H.-J. Boluijt, M. Seynen, and J. Zomerdijk for help with the design of the optical setup, the acquisition software, and the pulse integrator for power calibration. Furthermore, we are indebted to F. Bernal Arango for valuable insight in the calculations, C. Osorio, and E. Garnett for carefully checking the manuscript, and S. Rahimzadeh-Kalaleh Rodriguez and J. Gomez Rivas for discussion about luminescence in periodic particle systems. This work is part of the research program of the "Foundation for Fundamental Research on Matter (FOM)," which was financially supported by "The Netherlands Organization for Scientific Research (NWO).” A.F.K. gratefully acknowledges an NWO-Vidi grant for financial support. This work was furthermore supported by NanoNextNL, a microtechnology and nanotechnology consortium of the Government of the Netherlands and 130 partners.
[1] S. A. Maier, Plasmonics: Fundamentals and Applications (Springer, New York, 2007).

[2] M. Agio and A. Alu, Optical Antennas (Cambridge University Press, Cambridge, UK, 2013).

[3] P. Anger, P. Bharadwaj, and L. Novotny, Phys. Rev. Lett. 96, 113002 (2006).

[4] S. Kühn, U. Håkanson, L. Rogobete, and V. Sandoghdar, Phys. Rev. Lett. 97, 017402 (2006).

[5] H. Mertens, A. F. Koenderink, and A. Polman, Phys. Rev. B 76, 115123 (2007).

[6] V. Giannini, A. I. Fernandez-Dominguez, S. C. Heck, and S. A. Maier, Chem. Rev. 111, 3888 (2011).

[7] D. Punj, M. Mivelle, S. B. Moparthi, T. S. van Zanten, H. Rigneault, N. F. van Hulst, M. F. García-Parajo, and J. Wenger, Nat. Nanotechnol. 8, 512 (2013).
[8] A. Kinkhabwala, Z. Yu, S. Fan, Y. Avlasevich, K. Muellen, and W. E. Moerner, Nat. Photonics 3, 654 (2009).

[9] P. L. Stiles, J. A. Dieringer, N. C. Shah, and R. R. Van Duyne, Annu. Rev. Anal. Chem. 1, 601 (2008).

[10] F. Le, D. W. Brandl, Y. A. Urzhumov, H. Wang, J. Kundu, N. J. Halas, J. Aizpurua, and P. Nordlander, ACS Nano 2, 707 (2008).

[11] D. J. Bergman and M. I. Stockman, Phys. Rev. Lett. 90, 027402 (2003).

[12] M. I. Stockman, J. Opt. 12, 024004 (2010).

[13] M. A. Noginov, G. Zhu, A. M. Belgrave, R. Bakker, V. M. Shalaev, E. E. Narimanov, S. Stout, E. Herz, T. Suteewong, and U. Wiesner, Nature (London) 460, 1110 (2009).

[14] R. F. Oulton, V. J. Sorger, T. Zentgraf, R.-M. Ma, C. Gladden, L. Dai, G. Bartal, and X. Zhang, Nature (London) 461, 629 (2009). 
[15] R.-M. Ma, R. F. Oulton, V. J. Sorger, G. Bartal, and X. Zhang, Nat. Mater. 10, 110 (2011).

[16] S.-H. Kwon, J.-H. Kang, C. Seassal, S.-K. Kim, P. Regreny, Y.-H. Lee, C. M. Lieber, and H.-G. Park, Nano Lett. 10, 3679 (2010).

[17] Y.-J. Lu, J. Kim, H.-Y. Chen, C. Wu, N. Dabidian, C. E. Sanders, C.-Y. Wang, M.-Y. Lu, B.-H. Li, X. Qiu, W.-H. Chang, L.-J. Chen, G. Shvets, C.-K. Shih, and S. Gwo, Science 337, 450 (2012).

[18] M. T. Hill, Y.-S. Oei, B. Smalbrugge, Y. Zhu, T. De Vries, P. J. Van Veldhoven, F. W. M. Van Otten, T. J. Eijkemans, J. P. Turkiewicz, H. De Waardt, E. J. Geluk, S.-H. Kwon, Y.-H. Lee, R. Notzel, and M. K. Smit, Nat. Photonics 1, 589 (2007).

[19] M. Khajavikhan, A. Simic, M. Katz, J. H. Lee, B. Slutsky, A. Mizrahi, V. Lomakin, and Y. Fainman, Nature (London) 482, 204 (2012).

[20] J. Li, A. Salandrino, and N. Engheta, Phys. Rev. B 76, 245403 (2007).

[21] A. F. Koenderink, Nano Lett. 9, 4228 (2009).

[22] T. Kosako, Y. Kadoya, and H. F. Hofmann, Nat. Photonics 4, 312 (2010).

[23] A. G. Curto, G. Volpe, T. H. Taminiau, M. P. Kreuzer, R. Quidant, and N. F. van Hulst, Science 329, 930 (2010).

[24] F. B. Arango, A. Kwadrin, and A. F. Koenderink, ACS Nano 6, 10156 (2012).

[25] S. Zhang, D. A. Genov, Y. Wang, M. Liu, and X. Zhang, Phys. Rev. Lett. 101, 047401 (2008).

[26] B. Luk'yanchuk, N. I. Zheludev, S. A. Maier, N. J. Halas, P. Nordlander, H. Giessen, and C. T. Chong, Nat. Mater. 9, 707 (2010).

[27] N. Verellen, Y. Sonnefraud, H. Sobhani, F. Hao, V. V. Moshchalkov, P. Van Dorpe, P. Nordlander, and S. A. Maier, Nano Lett. 9, 1663 (2009).

[28] M. Hentschel, M. Saliba, R. Vogelgesang, H. Giessen, A. P. Alivisatos, and N. Liu, Nano Lett. 10, 2721 (2010).

[29] J. B. Lassiter, H. Sobhani, J. A. Fan, J. Kundu, F. Capasso, P. Nordlander, and N. J. Halas, Nano Lett. 10, 3184 (2010).

[30] G. Lozano, D. J. Louwers, S. R. K. Rodriguez, S. Murai, O. T. A. Jansen, M. A. Verschuuren, and J. G. Rivas, Light. Sci. Appl. 2, e66 (2013).

[31] S. Murai, M. A. Verschuuren, G. Lozano, G. Pirruccio, S. R. K. Rodriguez, and J. G. Rivas, Opt. Express 21, 4250 (2013).

[32] S. R. K. Rodriguez, S. Murai, M. A. Verschuuren, and J. G. Rivas, Phys. Rev. Lett. 109, 166803 (2012).

[33] G. Vecchi, V. Giannini, and J. Gómez Rivas, Phys. Rev. Lett. 102, 146807 (2009).

[34] C. L. Haynes, A. D. McFarland, L. L. Zhao, R. P. Van Duyne, G. C. Schatz, L. Gunnarsson, J. Prikulis, B. Kasemo, and M. Käll, J. Phys. Chem. B 107, 7337 (2003).

[35] S. L. Zou, N. Janel, and G. C. Schatz, J. Chem. Phys. 120, 10871 (2004).

[36] F. J. G. de Abajo, Rev. Mod. Phys. 79, 1267 (2007).

[37] R. de Waele, A. F. Koenderink, and A. Polman, Nano Lett. 7, 2004 (2007).

[38] J. Stehr, J. Crewett, F. Schindler, R. Sperling, G. von Plessen, U. Lemmer, J. M. Lupton, T. A. Klar, J. Feldmann, A. W. Holleitner, M. Forster, and U. Scherf, Adv. Mater. 15, 1726 (2003).

[39] F. van Beijnum, P. J. van Veldhoven, E. J. Geluk, M. J. A. de Dood, G. W. 't Hooft, and M. P. van Exter, Phys. Rev. Lett. 110, 206802 (2013).
[40] J. Y. Suh, C. H. Kim, W. Zhou, M. D. Huntington, D. T. Co, M. R. Wasielewski, and T. W. Odom, Nano Lett. 12, 5769 (2012).

[41] W. Zhou, M. Dridi, J. Y. Suh, C. H. Kim, D. T. Co, M. R. Wasielewski, G. C. Schatz, and T. W. Odom, Nat. Nanotechnol. 8, 506 (2013).

[42] I. Sersic, C. Tuambilangana, and A. F. Koenderink, New J. Phys. 13, 083019 (2011)

[43] M. A. Lieb, J. M. Zavislan, and L. Novotny, J. Opt. Soc. Am. B 21, 1210 (2004).

[44] N. Le Thomas, R. Houdre, M. V. Kotlyar, D. O'Brien, and T. F. Krauss, J. Opt. Soc. Am. B 24, 2964 (2007).

[45] S. Randhawa, M. Ujue Gonzalez, J. Renger, S. Enoch, and R. Quidant, Opt. Express 18, 14496 (2010).

[46] Y. Alaverdyan, E.-M. Hempe, A. N. Vamivakas, H. E. S. A Maier, and M. Atatüre, Appl. Phys. Lett. 94, 021112 (2009).

[47] C. Huang, A. Bouhelier, G. Colas des Francs, A. Bruyant, A. Guenot, E. Finot, J.-C. Weeber, and A. Dereux, Phys. Rev. B 78, 155407 (2008).

[48] T. H. Taminiau, S. Karaveli, N. F. van Hulst, and R. Zia, Nat Commun. 3, 979 (2012).

[49] G. A. Turnbull, P. Andrew, W. L. Barnes, and I. D. W. Samuel, Phys. Rev. B 67, 165107 (2003).

[50] L. Novotny and B. Hecht, Principles of Nano-Optics (Cambridge University Press, Cambridge, UK, 2007).

[51] H. P. Urbach and G. L. J. A. Rikken, Phys. Rev. A 57, 3913 (1998).

[52] H. Rigneault, F. Lemarchand, A. Sentenac, and H. Giovannini, Opt. Lett. 24, 148 (1999).

[53] W. A. Harrison, Solid State Theory (Dover, New York, 1970).

[54] M. Kretschmann and A. A. Maradudin, Phys. Rev. B 66, 245408 (2002).

[55] L. Langguth, D. Punj, J. Wenger, and A. F. Koenderink, ACS Nano 7, 8840 (2013).

[56] G. A. Turnbull, P. Andrew, M. J. Jory, W. L. Barnes, and I. D. W. Samuel, Phys. Rev. B 64, 125122 (2001).

[57] G. Heliotis, R. D. Xia, G. A. Turnbull, P. Andrew, W. L. Barnes, I. D. W. Samuel, and D. D. C. Bradley, Adv. Funct. Mater. 14, 91 (2004).

[58] S. Noda, J. Opt. Soc. Am. B 27, B1 (2010).

[59] S. Murai, Y. Tokuda, K. Fujita, and K. Tanaka, Appl. Phys. Lett. 101, 031117 (2012).

[60] W. L. Vos, R. Sprik, A. van Blaaderen, A. Imhof, A. Lagendijk, and G. H. Wegdam, Phys. Rev. B 53, 16231 (1996).

[61] Photonic Crystals and Light Localization in the 21st Century, edited by C. M. Soukoulis (Kluwer, Dordrecht, 2001), p. 194.

[62] H. Benisty and C. Weisbuch, Progress in Optics (Elsevier, Amsterdam, 2006), pp. 177-313.

[63] P. B. Johnson and P. W. Christy, Phys. Rev. B 6, 4370 (1972).

[64] J. J. Penninkhof, L. A. Sweatlock, A. Moroz, H. A. Atwater, A. van Blaaderen, and A. Polman, J. Appl. Phys. 103, 123105 (2008).

[65] V. Liu and S. Fan, Comput. Phys. Commun. 183, 2233 (2012).

[66] L. F. Li, J. Opt. Soc. Am. A 14, 2758 (1997).

[67] L. F. Li, J. Opt. Soc. Am. A 13, 1870 (1996).

[68] I. Sersic, M. Frimmer, E. Verhagen, and A. F. Koenderink, Phys. Rev. Lett. 103, 213902 (2009).

[69] N. I. Zheludev, S. L. Prosvirnin, N. Papasimakis, and V. A. Fedotov, Nat. Photonics 2, 351 (2008). 
[70] Z. V. Vardeny, A. Nahata, and A. Agrawal, Nat. Photonics 7, 177 (2013).

[71] A. Gopinath, S. V. Boriskina, N.-N. Feng, B. M. Reinhard, and L. Dal Negro, Nano Lett. 8, 2423 (2008).
[72] S. M. Lubin, A. J. Hryn, M. D. Huntington, C. J. Engel, and T. W. Odom, ACS Nano 7, 11035 (2013).

[73] E. Maciá, Rep. Prog. Phys. 75, 036502 (2012). 